\title{
Insecticide resistance in Anopheles arabiensis from Ethiopia (2012-2016): a nationwide study for insecticide resistance monitoring
}

Louisa A. Messenger ${ }^{1 *}$, Josephat Shililu², Seth R. Irish', Gedeon Yohannes Anshebo², Alemayehu Getachew Tesfaye ${ }^{2}$, Yemane Ye-Ebiyo ${ }^{2}$, Sheleme Chibsa ${ }^{3}$, Dereje Dengela ${ }^{4}$, Gunawardena Dissanayake ${ }^{3}$,Estifanos Kebede ${ }^{5}$, Endalew Zemene ${ }^{5}$, Abebe Asale ${ }^{5}$, Mekonnen Yohannes ${ }^{6}$, Hiwot Solomon Taffese ${ }^{7}$, Kristen George ${ }^{8}$, Christen Fornadel ${ }^{8}$, Aklilu Seyoum ${ }^{4}$, Robert A. Wirtz ${ }^{1}$ and Delenasaw Yewhalaw ${ }^{5,9}$

\begin{abstract}
Background: Indoor residual spraying (IRS) and long-lasting insecticidal nets (LLINS) remain the cornerstones of malaria vector control. However, the development of insecticide resistance and its implications for operational failure of preventative strategies are of concern. The aim of this study was to characterize insecticide resistance among Anopheles arabiensis populations in Ethiopia and describe temporal and spatial patterns of resistance between 2012 and 2016.

Methods: Between 2012 and 2016, resistance status of An. arabiensis was assessed annually during the long rainy seasons in study sites from seven of the nine regions in Ethiopia. Insecticide resistance levels were measured with WHO susceptibility tests and CDC bottle bioassays using insecticides from four chemical classes (organochlorines, pyrethroids, organophosphates and carbamates), with minor variations in insecticides tested and assays conducted between years. In selected sites, CDC synergist assays were performed by pre-exposing mosquitoes to piperonyl butoxide (PBO). In 2015 and 2016, mosquitoes from DDT and deltamethrin bioassays were randomly selected, identified to species-level and screened for knockdown resistance ( $k d r)$ by PCR.

Results: Intense resistance to DDT and pyrethroids was pervasive across Ethiopia, consistent with historic use of DDT for IRS and concomitant increases in insecticide-treated net coverage over the last 15 years. Longitudinal resistance trends to malathion, bendiocarb, propoxur and pirimiphos-methyl corresponded to shifts in the national insecticide policy. By 2016, resistance to the latter two insecticides had emerged, with the potential to jeopardize future longterm effectiveness of vector control activities in these areas. Between 2015 and 2016, the West African (L1014F) kdr allele was detected in $74.1 \%(n=686 / 926)$ of specimens, with frequencies ranging from 31 to $100 \%$ and 33 to $100 \%$ in survivors from DDT and deltamethrin bioassays, respectively. Restoration of mosquito susceptibility, following preexposure to PBO, along with a lack of association between $k d r$ allele frequency and An. arabiensis mortality rate, both indicate metabolic and target-site mutation mechanisms are contributing to insecticide resistance.
\end{abstract}

\footnotetext{
*Correspondence: ndx1@cdc.gov

1 Entomology Branch, Centers for Disease Control and Prevention, 1600

Clifton Road, Atlanta, GA 30329-4027, USA

Full list of author information is available at the end of the article
} 
Conclusions: Data generated by this study will strengthen the National Malaria Control Programme's insecticide resistance management strategy to safeguard continued efficacy of IRS and other malaria control methods in Ethiopia.

Keywords: Insecticide resistance, Anopheles arabiensis, Resistance mechanisms, Intensity assays, Malaria, kdr, Ethiopia

\section{Background}

Despite the scaling-up of key diagnostic, treatment and preventative measures, malaria remains a considerable public health problem in Ethiopia, with over 50.6 million (60\% of the total population) at significant risk [1]. Transmission of Plasmodium falciparum and Plasmodium vivax is highly heterogeneous and unstable across the country, concentrated in lowland and highland fringe areas [2]. Unlike other sub-Saharan countries, where malaria morbidity and mortality mainly impacts young children, in Ethiopia, low levels of immunity predispose many individuals to clinically severe malaria and epidemics among all age groups. As part of the National Malaria Strategic Plan (2014-2020), vector control by the National Malaria Control Programme (NMCP), with support from the President's Malaria Initiative (PMI) and the Global Fund, is based on indoor residual spraying (IRS) and universal coverage campaigns of long-lasting insecticidal nets (LLINs) [1-4].

IRS was first implemented in Ethiopia in 1959 and continues to play a prominent role in malaria control. LLIN coverage has been scaled up since 2005, resulting in over 64 million nets distributed by 2014 [2]. However, the long-term effectiveness of both strategies is currently under threat from widespread emergence of insecticide resistance in the principal malaria vector, Anopheles arabiensis [3]. To date, in Ethiopia, An. arabiensis has developed resistance against insecticides belonging to all four chemical classes approved for IRS, including DDT (organochlorine), malathion (organophosphate), bendiocarb and propoxur (carbamates) and alpha-cypermethrin, cyfluthrin, deltamethrin, etofenprox, lambda-cyhalothrin and permethrin (pyrethroids) [5-14]. The West African $k d r$ (L1014F) mutation has been reported in An. arabiensis populations at high frequencies $[9,14,15]$ and preexposure of An. arabiensis to piperonyl butoxide (PBO) significantly increased vector susceptibility to deltamethrin and permethrin [12], suggesting both metabolic and target-site mutation mechanisms are responsible for insecticide resistance. Historically, DDT, and to a lesser extent, malathion were used for IRS in Ethiopia [12]. In 2010, vector control by the NMCP, with support from PMI, discontinued DDT spraying in favour of deltamethrin, which was used initially in combination with bendiocarb from 2011, before being superseded exclusively by bendiocarb and propoxur in 2013. In 2015, in response to incipient resistance, PMI-supported IRS activities were based on bendiocarb in 28 districts (and focal pirimiphosmethyl application in 8 districts) and in 2016, pirimiphosmethyl replaced bendiocarb in all PMI-supported districts [2] (Fig. 1 and Additional file 1: Table S1).

Considering only a limited number of alternate insecticides are available for public health use, the aim of this study was to characterize contemporary nationwide insecticide resistance in An. arabiensis populations and describe longitudinal trends in resistance between 2012 and 2016, to strengthen the NMCP's insecticide resistance monitoring and management strategy and safeguard continued efficacy of IRS in Ethiopia [16].

\section{Methods}

\section{Study sites}

Data are from study sites in seven of the nine regions [Afar, Amhara, Benishangul Gumuz, Gambela, Oromia, Southern Nations, Nationalities, and Peoples' Region (SNNPR), and Tigray] in Ethiopia between 2012 and 2016.

\section{Susceptibility tests}

For all susceptibility tests, mosquito larvae were collected by dipping from a range of breeding sites in each study area and reared to adults under standard insectary conditions (temperature $25 \pm 2{ }^{\circ} \mathrm{C}$, relative humidity $80 \pm 10 \%$ ). Bioassays were conducted annually during the long rainy season (June-September). WHO tube tests and CDC bottle bioassays were used to determine susceptibility levels of Anopheles gambiae s.l. populations (henceforth An. arabiensis) to different insecticides, with minor variations in insecticides tested and assays conducted between years (Additional file 1: Table S2). From 2014 onwards, CDC resistance intensity assays were undertaken and synergist assays were also performed by pre-exposing mosquitoes to $\mathrm{PBO}$ in selected sites. For all assays, care was taken during storage and field transportation of insecticide-impregnated papers and technical grade insecticide stock solutions to reduce heat exposure and minimize potential loss of efficacy.

\section{WHO susceptibility tests}

World Health Organization (WHO) susceptibility tests for the following eleven insecticides with diagnostic doses (alpha-cypermethrin $(0.05 \%)$, bendiocarb $(0.1 \%)$, 


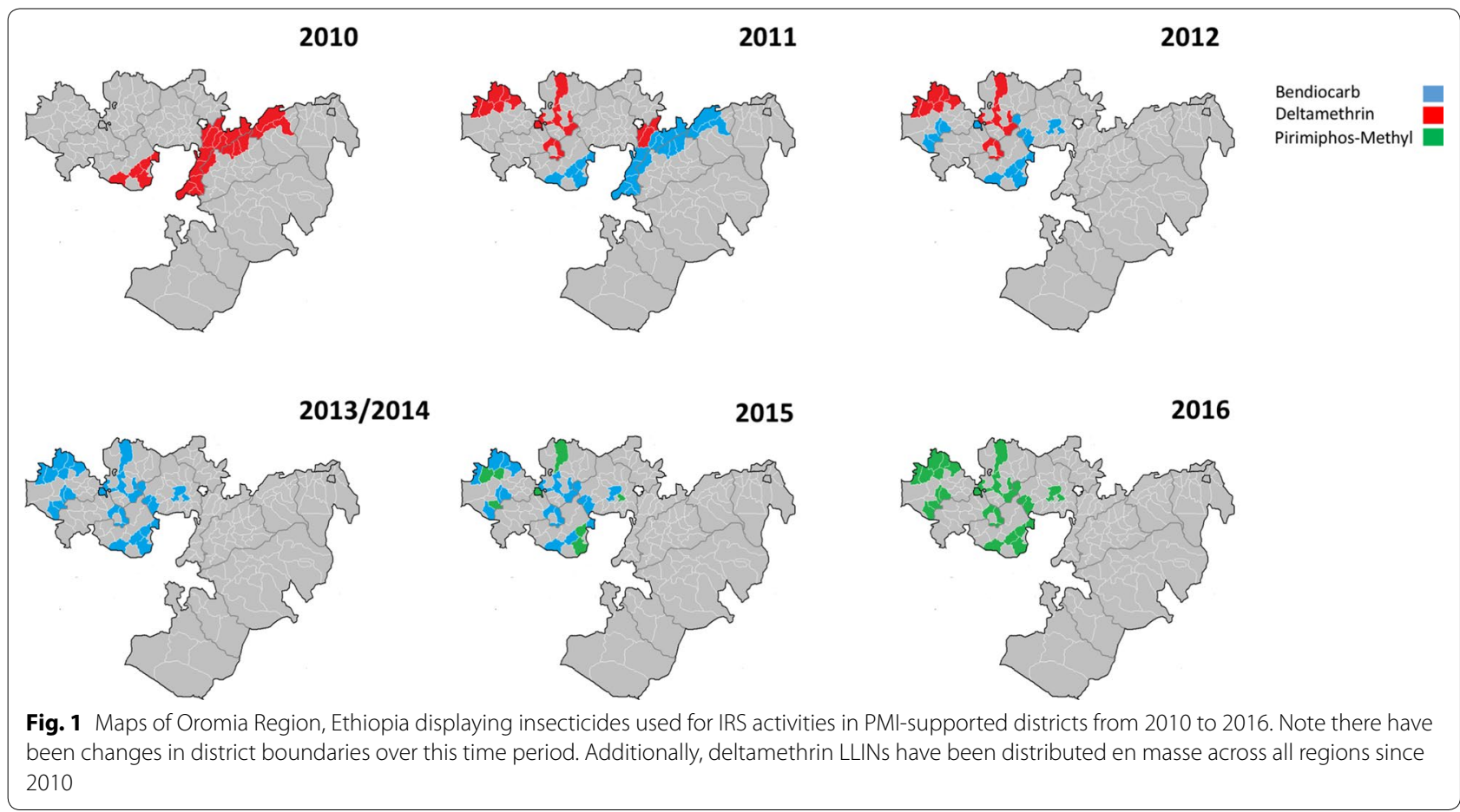

DDT (4\%), deltamethrin (0.05\%), etofenprox (0.5\%), fenitrothion (1\%), lambda-cyhalothrin (0.05\%), malathion (5\%), permethrin $(0.75 \%)$, pirimiphos-methyl $(0.25 \%)$ and propoxur $(0.1 \%)$ ) were conducted according to WHO guidelines [17]. The diagnostic dose of insecticides used on papers is generally twice the $\mathrm{LC}_{99}$ values systematically determined from baseline studies in multiple locations [18]. In 2012, four replicates of 20-25 non-blood fed, 2-3 days old adult female mosquitoes were exposed to different insecticide-impregnated papers in WHO tubes for $1 \mathrm{~h}$ (except in the case of fenitrothion where mosquitoes were exposed for $2 \mathrm{~h}$ ) and in parallel, one replicate of control mosquitoes (20-25 mosquitoes per tube) was exposed to oil-impregnated papers; from 2013 onwards, two control replicates using 25 mosquitoes were performed. For all assays, mosquito mortality was recorded after a 24-h holding period. Cotton wool soaked in $10 \%$ sugar solution was provided to mosquitoes on top of the holding tube and optimum temperature and relative humidity was maintained using a damp towel placed on top of boxes where holding tubes were kept.

\section{CDC bottle bioassays}

CDC bottle bioassays for seven insecticides (alphacypermethrin, bendiocarb, DDT, deltamethrin, lambdacyhalothrin, permethrin and propoxur) were conducted according to published guidelines [19]. Stock solutions were prepared by diluting technical grade insecticide in $50 \mathrm{~mL}$ of acetone. Each Wheaton $250 \mathrm{~mL}$ bottle along with its cap was coated with $1 \mathrm{~mL}$ of stock solution $(12.5 \mu \mathrm{g} /$ bottle for alpha-cypermethrin, bendiocarb, deltamethrin, lambda-cyhalothrin and propoxur, $100 \mu \mathrm{g} /$ bottle for DDT and $21.5 \mu \mathrm{g} /$ bottle for permethrin) by rolling and inverting the bottles. In each test, a control bottle was coated with $1 \mathrm{~mL}$ of acetone. Following coating, bottles were covered with mats and left to dry. Approximately, 10-25 non-blood fed, 2-3 days old adult female mosquitoes were introduced into each bottle using a mouth aspirator and mortality was recorded at $15 \mathrm{~min}$ intervals up to $30 \mathrm{~min}$ for all insecticides except DDT; for this assay mosquitoes were exposed for $45 \mathrm{~min}$. From 2014 onwards, CDC resistance intensity assays were performed by testing $1,2,5$ and 10 times the concentration required to kill all mosquitoes $\left(\mathrm{LC}_{100}\right)$, as determined in a series of baseline experiments [19], and in selected sites, synergist assays were also conducted by pre-exposing mosquitoes to PBO for $1 \mathrm{~h}(100 \mu \mathrm{g} / \mathrm{bottle})$.

\section{Molecular identification of Anopheles gambiae species complex}

Mosquitoes used in bioassay tests were identified morphologically using standard keys [20]. In 2015 and 2016, sub-samples of both surviving and dead mosquitoes from WHO tests were randomly selected by insecticide (DDT and deltamethrin), site and region for molecular species identification and $k d r$ allele detection at the Molecular Biology Laboratory, Tropical and Infectious Diseases Research Centre (TIDRC) of Jimma University. Genomic 
DNA was extracted following the procedure described by Collins et al. [21]. DNA was re-suspended in $25 \mathrm{ml}$ sterile TE-buffer (10 mM Tris-HCl pH 8, 1 mM EDTA). Molecular identification of An. gambiae s.l. was carried out by species-specific PCR using primers for An. gambiae s.s., An. arabiensis and Anopheles quadriannulatus species B (Anopheles amharicus) according to Scott et al. [22], with modifications $[9,23]$. Briefly, genomic DNA was mixed with primers AR (5'-AAGTGTCCTTCTCCATCCRA-3'; specific for An. arabiensis), AG (5'-CTGGTTTGGTCGGCACGTTT-3; specific for An. gambiae s.s.), QD-b (5'-AGTGTCCAATGTCTGTGAAG-3'; specific for $A n$. quadriannulatus species B) and UN (5'-GTGTGCCCCTTCCTCGATGT-3'; common for all species) in a $25 \mu \mathrm{L}$ reaction. Amplification reactions contained $1 \mu \mathrm{L}$ of DNA, $1.5 \mathrm{mM} \mathrm{MgCl}_{2}, 10 \mathrm{mM}$ Tris- $\mathrm{HCl}$ (pH 8.4), $50 \mathrm{mM}$ $\mathrm{KCl}, 0.1 \%$ Triton X-100, $200 \mu \mathrm{M}$ of dNTPs (Amersham, Buckinghamshire, United Kingdom), 25 pmol of primers AR, AG, QD-b and UN and $0.25 \mathrm{U}$ of SilverStar DNA polymerase (Eurogentec, Seraing, Belgium) [24]. PCR reaction conditions are described in Scott et al. [22]. Amplified PCR products were visualized on $2 \%$ agarose gels, stained with ethidium bromide. An. arabiensis strain from the Sekoru colony, maintained at the Vector Biology and Control Research Unit, TIDRC, Jimma University, was used as a positive control.

\section{Detection of resistance mutations}

West African $k d r$ (L1014S) and East African $k d r$ (L1014F) alleles were detected using adapted protocols [24] for allele-specific PCR (AS-PCR), developed by Martinez-Torres et al. [25] and Ranson et al. [26]. Primers Agd1 (5'-ATAGATTCCCCGACCATG-3'), Agd2 (5'-AGACAAGGATGATGAACC-3'), Agd3 (5'-AATTTGCATTACTTACGACA-3') and Agd4 (5'-CTGTAGTGATAGGAAATTTA- $3^{\prime}$ ) were used to detect the L1014F allele (AS-PCR Agd3), whereas primers Agd1, Agd2, Agd4 and Agd5 (5'-TTTGCATTACTTACGACTG-3') were used to detect the L1014S allele (AS-PCR Agd5). Amplifications were performed in $50 \mu \mathrm{L}$ reactions containing $2 \mu \mathrm{L}$ DNA, $1 \times$ Qiagen PCR buffer, $0.5 \mathrm{mM}$ $\mathrm{MgCl}_{2}, 100 \mathrm{nM}$ of each primer, $200 \mu \mathrm{M}$ dNTPs, and $1 \mathrm{U}$ Taq DNA polymerase (Taq PCR core kit, Qiagen, Hilden, Germany). The cycling conditions were: initial $94{ }^{\circ} \mathrm{C}$ denaturation for $5 \mathrm{~min}, 10$ cycles of 1 min denaturation at $94{ }^{\circ} \mathrm{C}, 30 \mathrm{~s}$ annealing at $54{ }^{\circ} \mathrm{C}$ and $30 \mathrm{~s}$ extension at $72{ }^{\circ} \mathrm{C}$, followed by 30 cycles of $1 \mathrm{~min}$ denaturation at $94{ }^{\circ} \mathrm{C}, 30 \mathrm{~s}$ annealing at $47^{\circ} \mathrm{C}$ and $30 \mathrm{~s}$ extension at $72{ }^{\circ} \mathrm{C}$, and a final extension at $72{ }^{\circ} \mathrm{C}$ for $10 \mathrm{~min}$. Amplification products were visualized on $2 \%$ agarose gels, stained with ethidium bromide. An. arabiensis from the Sekoru colony (a $k d r$ negative mosquito strain) was used as a negative control.

\section{Data analysis}

Data were interpreted according to the $\mathrm{WHO}$ guidelines [17]; mortality of $98 \%$ or higher in susceptibility tests indicates susceptibility, mortality of $90-97 \%$ is suggestive of resistance and mortality of less than 90\% indicates resistance. Mortality was corrected using Abbott's formula, when mortality in control assays was between 5 and 20\% [17, 27]. A bioassay was repeated if control mortality exceeded $20 \%$. Per site, mean percent mosquito mortality was determined across all replicates for a given insecticide. Pearson's Chi squared tests were used to evaluate the association of $k d r$ frequency with WHO assay results and to test for deviations from Hardy-Weinberg equilibrium. Cohen's Kappa ( $\kappa$ ) was calculated to quantify the magnitude of agreement between WHO susceptibility tests and CDC bottle bioassays [28]; values were interpreted as poor $(\kappa \leq 0)$, slight $(0<\kappa \leq 0.2)$, fair $(0.2<\kappa \leq 0.4)$, moderate $(0.4<\kappa \leq 0.6)$, substantial $(0.6<\mathrm{\kappa} \leq 0.8)$ and almost perfect agreement $(0.8<\kappa \leq 1.0)$ [29]. All statistical analyses were performed in Stata/IC 14.2 (Stata Corp., College Station, USA) with the level of significance set at $\alpha=0.05$.

\section{Results \\ WHO susceptibility tests}

Results from WHO susceptibility tests conducted in eight sentinel sites (Alamata, Amibara, Asendabo, Bahir Dar, Chewaka, Halaba, Lare and Ziway-Dugda) between 2012 and 2016 are summarized in Table 1 and Fig. 2. WHO tests conducted in additional areas from 2013 onwards are detailed in Additional file 1: Tables S3-S6 by year.

In 2012, high levels of pyrethroid resistance were evident across Ethiopia, with An. arabiensis mortality levels of $50 \%$ or less for alpha-cypermethrin, deltamethrin, etofenprox, lambda-cyhalothrin and permethrin. Similarly, all mosquito populations were highly resistant to DDT (mortality ranging from 0 to $13 \%$ ) and demonstrated variable levels of susceptibility to the organophosphate malathion (26-90\% mortality) (Table 1). In contrast, $A n$. arabiensis was fully susceptible to organophosphates fenitrothion and pirimiphos-methyl and the carbamate propoxur, with the exception of Chewaka, where average mortality for the latter was 96\%. Low levels of developing bendiocarb (carbamate) resistance were detected in three study sites, Asendabo, Bahir Dar and Chewaka (mortality of 93,87 and $90 \%$, respectively).

In 2015-2016, most An. arabiensis populations presented consistent susceptibility profiles, with minor variations in some resistance levels; notably in four sites (Asendabo, Chewaka, Halaba and Ziway-Dugda) susceptibility to malathion increased over time (from 66 to 83\%, 58 to $96 \%, 48$ to $96 \%$ and 90 to $98 \%$ average mosquito mortality, respectively), while mosquito mortality to 


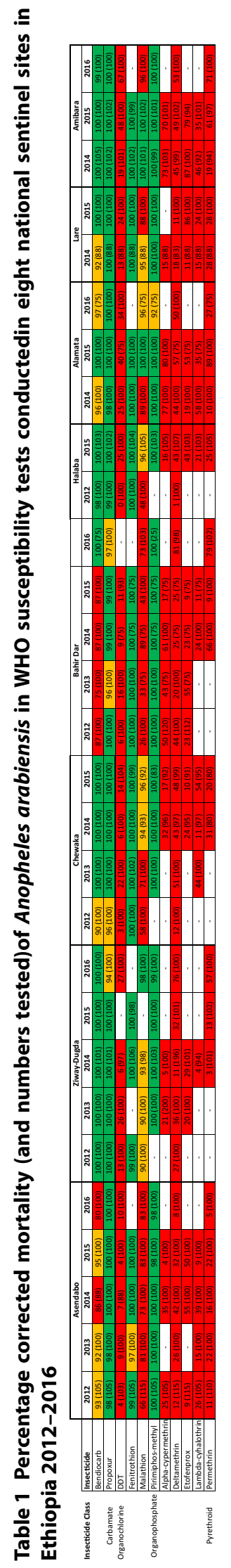




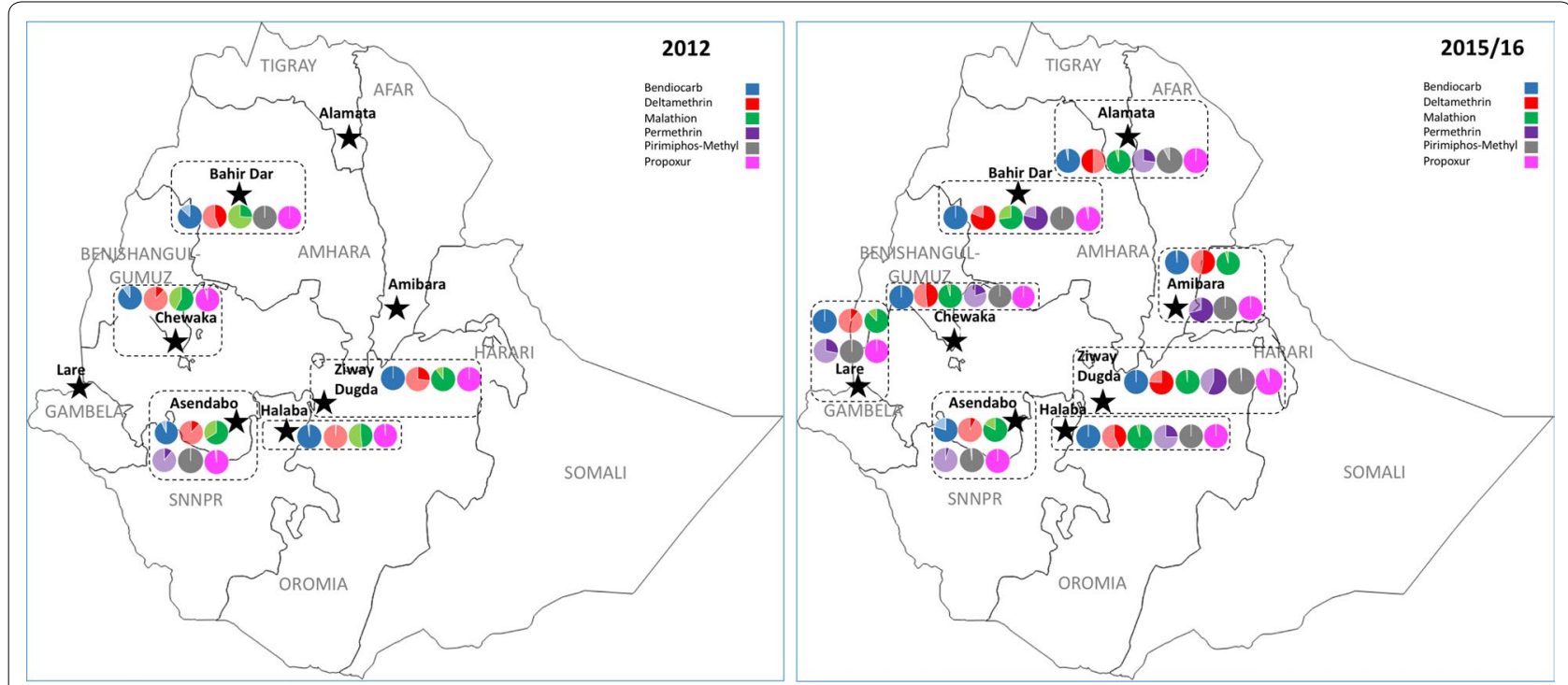

Fig. 2 Maps of Ethiopia displaying trends in An. arabiensis susceptibility levels to bendiocarb, deltamethrin, malathion, permethrin, pirimiphosmethyl and propoxur, as measured using WHO susceptibility tests, between 2012 (left) and 2015/16 (right). Vector populations were sampled from eight sentinel sites (Alamata, Amibara, Asendabo, Bahir Dar, Chewaka, Halaba, Lare and Ziway Dugda) in six regions. Legend colours indicate the insecticide under evaluation, with darker shading denoting average mosquito mortality

pirimiphos-methyl began to decline slightly in Asendabo and Ziway-Dugda (from 100 to $98 \%$ and 100 to $99 \%$ mortality, respectively) and possible resistance to propoxur emerged in Ziway-Dugda (94\% mortality) and Bahir Dar (97\% mortality). Resistance to pirimiphos-methyl and propoxur was also detected in additional study sites from Oromia region; An. arabiensis mortality was $85 \%$ in Babile in 2016 to pirimiphos-methyl, and $75 \%$ and $95 \%$ to propoxur in Abaya in 2013 and in Nono in 2016, respectively (Additional file 1: Tables S3, S6). Interestingly, vectors from an area of sesame cultivation sampled in 2016 (Metema; Amhara Region) demonstrated almost complete susceptibility to deltamethrin (average mortality of 99\%; Additional file 1: Table S6).

Similar longitudinal trends were observed in Alamata, Lare and Amibara, where resistance monitoring began in 2014 (Table 1). In these areas, at baseline, vector populations were also resistant to pyrethroids and DDT, fully susceptible to fenitrothion, pirimiphos-methyl and propoxur and potentially resistant to bendiocarb in Alamata and Lare (mortality of 96 and 92\%, respectively). In these latter two sites, resistance to malathion was also detected (mortality of 89 and 95\%, respectively). By 2016, putative resistance to pirimiphos-methyl had developed in Alamata (mortality of 92\%) and to malathion in Amibara (mortality of 96\%). On a yearly basis, levels of resistance fluctuated within sites, in some cases modestly (e.g. in Asendabo, mortality to bendiocarb oscillated from 93\% in 2012 , to $86 \%$ in $2014,95 \%$ in 2015 and $80 \%$ in 2016 ) and in others more dramatically, beyond what might be expected of stochastic variation (e.g. in Bahir Dar, mortality to malathion ranged from $26 \%$ in 2012 , to $89 \%$ in 2014 and $43 \%$ in 2015).

\section{CDC bottle bioassays}

From 2013 onwards, CDC bottle bioassays, resistance intensity and synergist assays were conducted in additional study sites, which differed between years (Additional file 1: Tables S7-S10). Data from Ziway-Dugda, where these tests were performed routinely throughout the monitoring period are presented in Fig. 3 and Additional file 1: Table S11, in comparison with concurrent WHO bioassays. Results from both WHO tests (diagnostic dose) and CDC bottle bioassays (2X) were concordant for bendiocarb and propoxur; vector populations were fully susceptible to both insecticides until 2016. However, levels of pyrethroid resistance were not comparable between techniques, e.g. An. arabiensis mortality in 2014 to deltamethrin was $11 \%$ in WHO tests, compared to $70 \%$ in CDC bottle bioassays at the same discriminatory dose $(\kappa \leq 0$, for all comparisons between pyrethroid assays in 2014, 2015 and 2016).

While consistently high levels of pyrethroid resistance were observed using WHO tests, examination of CDC bioassays from additional study areas identified some completely susceptible $A n$. arabiensis populations, e.g. mortality was $100 \%$ to deltamethrin in Abedogoro at the equivalent WHO diagnostic dose (2X) in 2015. Focal 


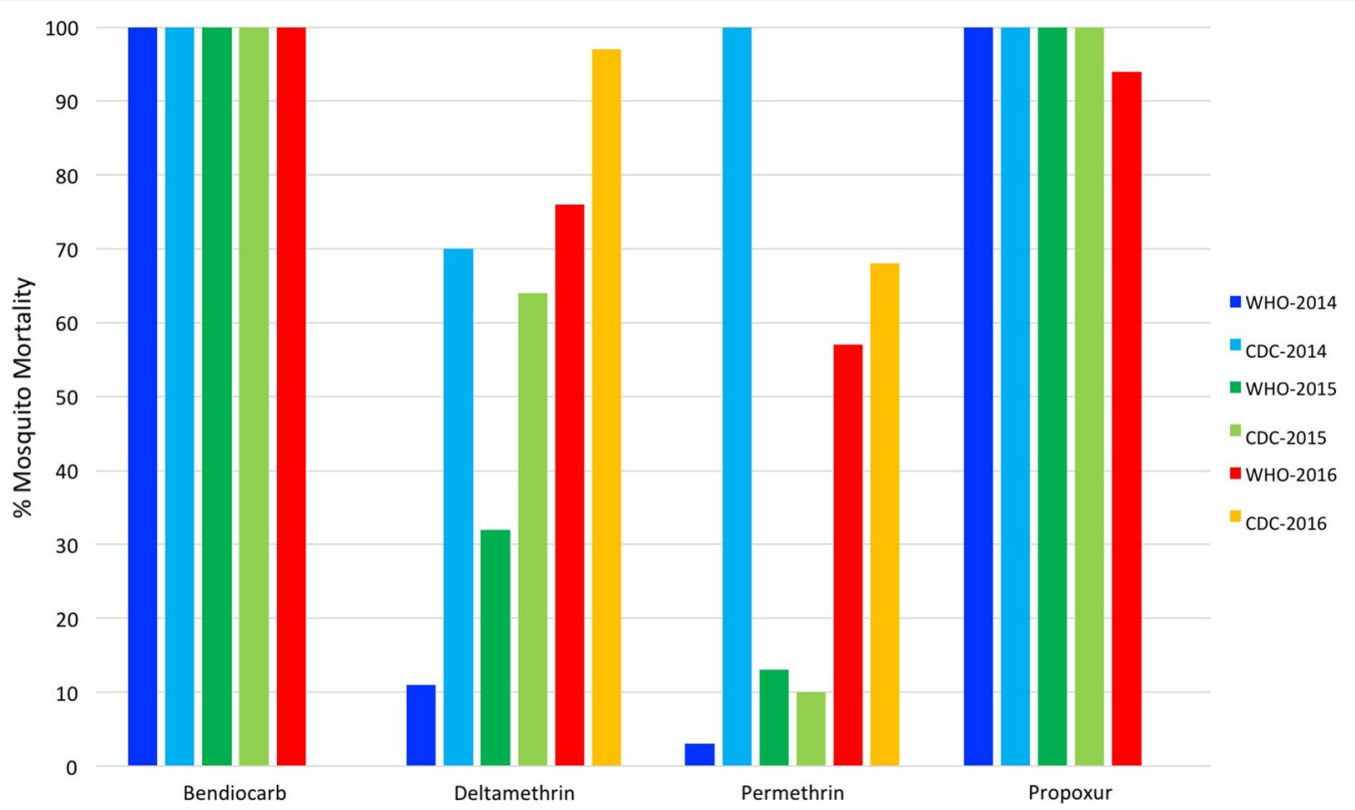

Fig. 3 Comparison of WHO and CDC bottle bioassays (at both respective diagnostic doses), conducted using bendiocarb, deltamethrin, permethrin and propoxur in Ziway-Dugda from 2014 to 2016

patterns of pyrethroid resistance were also apparent in 2013, where vector populations, susceptible to one or more pyrethroids, were identified in eleven areas across three regions (Additional file 1: Table S7). In these sites resistance to a particular pyrethroid was not necessarily associated with increased tolerance to another, e.g. in Gobu-Seyo (Oromia Region) mosquito mortality was $76,49,98$ and $8 \%$ to alpha-cypermethrin, deltamethrin, lambda-cyhalothrin and permethrin at the CDC diagnostic doses, respectively. In other areas with high levels of pyrethroid resistance, CDC resistance intensity assays detected some mosquitoes capable of surviving ten times the diagnostic dose of deltamethrin or permethrin (e.g. average mortality of 75 and 65\% in Asendabo in 2015, respectively; Additional file 1: Table S9). High intensities of DDT resistance were also observed in these areas, e.g. $95 \%$ of mosquitoes survived ten times the diagnostic dose in Wondogenet in 2014 (Additional file 1: Table S8).

In all sites where mosquitoes were pre-exposed to PBO, a synergist that interferes with oxidase activity, resistance to both deltamethrin and permethrin was reduced, increasing mortality to $87-100 \%$ when mosquitoes were exposed to the 1X dose (Additional file 1: Tables S8-S11).

\section{Molecular detection of species and resistance mutations} Following WHO susceptibility tests in 2015, three hundred and sixty-four surviving and dead An. gambiae s.l. specimens were randomly selected from eight study sites in six regions [Asendabo, Chewaka, Ziway-Dugda (Oromia region), Bahir Dar (Amhara region), Amibara (Afar region), Alamata (Tigray region), Halaba (SNNPR) and Abobo (Gambela region)] and were assayed to determine species. Of these, $94.8 \%(345 / 364)$ of samples amplified and were identified as An. arabiensis. Non-amplifiers were evenly distributed across all study sites, except Amibara and Ziway-Dugda where all specimens were successfully classified. In 2016, six hundred and sixty-two An. gambiae s.l. were collected from ten study sites in six regions: Asendabo, Babile, Nono and Ziway-Dugda (Oromia region), Metema (Amhara region), Amibara (Afar region), Abobo (Gambela region), Alamata and Humera (Tigray region) and Arba Minch (SNNPR), and 86.7\% (574/662) of specimens were confirmed as An. arabiensis. Higher proportions of non-amplifiers were collected from Metema (17.6\% of total mosquitoes sampled), Nono (30\%), Arba Minch and Abobo (both 17.5\%), than other neighboring areas (range of remaining sites: 5-12.5\%).

In 2015, the presence of $k d r$ was also assessed in matched mosquito samples (Table 2). The West African (L1014F) $k d r$ allele was identified in $75.5 \%(\mathrm{n}=275 / 364)$ of specimens ( 215 alive and 60 dead), with allele frequencies ranging from 50 to $100 \%$ and 13 to $88 \%$ in surviving and dead An. arabiensis from DDT bioassays, respectively, and from 36 to $100 \%$ and 13 to $100 \%$ in surviving and dead $A n$. arabiensis from deltamethrin bioassays, respectively. The majority of vectors surviving bioassays were homozygous for $k d r(54.0 \%$; $116 / 215)$ compared to those 


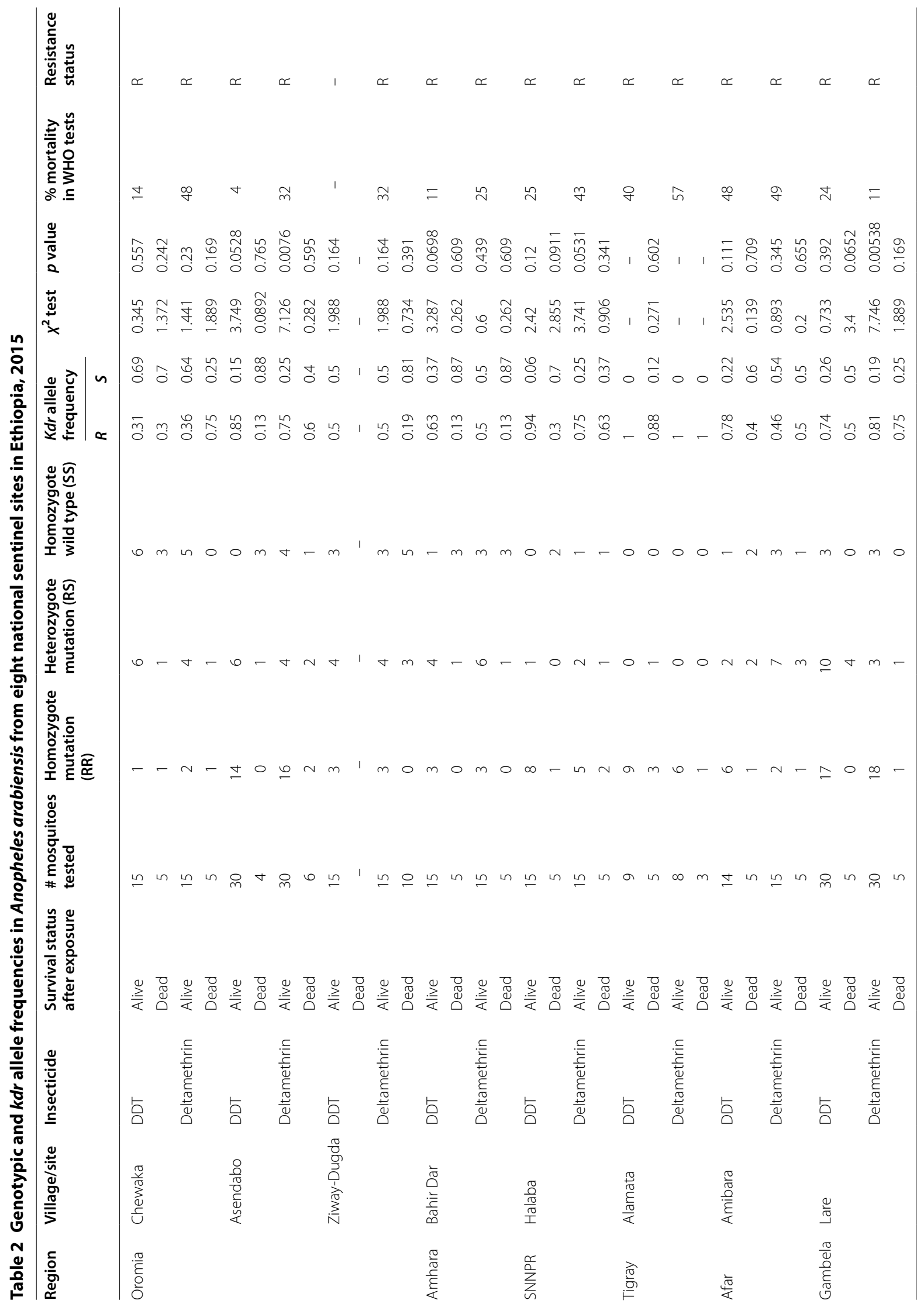


that died (23.3\%; 14/60); $29.3 \%(63 / 215)$ and 36.7\% (22/60) were heterozygous for $k d r$, respectively. The $k d r$-West allele was present in $100 \%$ of surviving vectors from one study site (Alamata; Tigray region) and $94 \%$ in another (Halaba; the SNNPR). Deviations from Hardy-Weinberg equilibrium were only observed in An. arabiensis survivors of deltamethrin bioassays in Asendabo and Lare $\left(\chi^{2}=7.13 ; p=0.0076\right.$ and $\chi^{2}=7.75 ; p=0.0054$ respectively) (Table 2). There was no significant association between $k d r$ allele frequency and An. arabiensis mortality following exposure to DDT or deltamethrin $(p=0.227$ and $p=0.208$, respectively). The East African (L1014S) $k d r$ allele was not detected in any samples assayed.

In 2016, all mosquito specimens confirmed as An. arabiensis $(\mathrm{n}=562$; with the exception of 8 and 4 samples from Metema and Alamata, respectively) were assayed for $k d r$ (Table 3). L1014F $k d r$ was detected in $73.5 \%$ ( $\mathrm{n}=413 / 562)$ of An. arabiensis (322 alive and $91 \mathrm{dead}$ ), with allele frequencies ranging from 31 to $89 \%$ and 10 to $100 \%$ in surviving and dead mosquitoes from DDT bioassays, respectively, and from 33 to $100 \%$ and 6 to $94 \%$ in surviving and dead mosquitoes from deltamethrin bioassays, respectively. There was no significant differences in $k d r$ allele frequency between years among DDT or deltamethrin bioassay survivors $(p=0.229$ and $p=0.158$, respectively). As previously, the majority of An. arabiensis surviving bioassays were homozygous for $k d r(42.2 \%$; $136 / 322)$ compared to those that died $(28.6 \%$; $26 / 91)$; $33.2 \%(107 / 322)$ and $28.6 \%(26 / 91)$ were heterozygous for $k d r$, respectively. The $k d r$-West allele was fixed in surviving vectors from two study sites (Babile and Nono; Oromia region); in Alamata, $k d r$ frequencies had declined from $100 \%$ in 2015 to $62 \%$ and $57 \%$ among survivors of DDT and deltamethrin bioassays, respectively. Consistent with results from 2015, deviations from Hardy-Weinberg equilibrium were identified among $A n$. arabiensis in Asendabo $\left(x^{2}=9.71 ; p=0.0018\right.$ for deltamethrin survivors) (Table 3 ) and in additional vector populations in Abobo $\left(\mathrm{X}^{2}=13.60 ; p=0.00023, \mathrm{X}^{2}=3.83\right.$; $p=0.05$ for survivors of DDT and deltamethrin bioassays, respectively), Arba Minch $\left(\chi^{2}=4.87 ; p=0.027\right.$ for DDT survivors), Alamata ( $x^{2}=4.83 ; p=0.028$ for deltamethrin survivors) and Amibara $\left(\chi^{2}=5.62 ; p=0.018\right.$ and $\chi^{2}=6.53 ; p=0.011$ for DDT and deltamethrin survivors, respectively). There was no significant association between $k d r$ allele frequency and An. arabiensis mortality following exposure to DDT or deltamethrin $(p=0.231$ and $p=0.267$, respectively).

\section{Discussion}

The development of pervasive insecticide resistance across sub-Saharan Africa threatens to jeopardize the long-term effectiveness of both IRS and LLINs for malaria control [30, 31]. This study presents data from the largest nationwide, longitudinal monitoring of insecticide resistance among An. arabiensis populations to four classes of insecticides in Ethiopia. Intense resistance to pyrethroids (alpha-cypermethrin, deltamethrin, etofenprox, lambda-cyhalothrin and permethrin) and DDT were commonplace, and in many sites, vectors were able to survive exposure to five to ten times the diagnostic dose. These high levels of resistance are likely a direct consequence of historic DDT use for IRS, as well as its considerable application between 2000 and 2005, where $255,000-298,000 \mathrm{~kg} /$ year were used [32], alongside mass distributions of LLINs [2].

Patterns of resistance to bendiocarb, malathion, propoxur and pirimiphos-methyl also corresponded to shifts in the national insecticide policy [2]. Since 2012, $A n$. arabiensis susceptibility to malathion increased in some areas, potentially attributable to the discontinuation of this insecticide for IRS; malathion was last used extensively for malaria control from 2003 to 2005 by the NMCP in areas with reported DDT resistance [5, 9]. Between 2011 and 2015, bendiocarb (with deltamethrin in 2011-2012) was the insecticide of choice for PMI-supported IRS activities in Oromia Region, where low levels of mosquito resistance were initially detected in 2012. However, because bendiocarb was largely abandoned due to its short residual efficacy, relative to other organophosphates and carbamates [33], only moderate levels of resistance developed in a few areas. Concurrent propoxur spraying in 2012 was accompanied by the emergence of potential resistance in some An. arabiensis populations by 2014 and likewise, decreased An. arabiensis susceptibility to pirimiphos-methyl was observed in 2016, concomitant with the switch to this insecticide in selected districts in 2015; however it should be noted that these sites fell outside the districts where pirimiphosmethyl was used for IRS. In general, the reactive, and in some cases, heterogeneous use of different insecticides has resulted in highly focal, volatile resistance profiles across sentinel sites [34], complicating the prospective deployment of interventions for vector control. Study observations are consistent with earlier cross-sectional evaluations from Ethiopia [35], which also describe widespread resistance to DDT and pyrethroids, as well as more restricted decreases in An. arabiensis susceptibility to malathion, bendiocarb and propoxur [5-15]. Furthermore, results from Sudan, Kenya and Eritrea corroborate large-scale resistance trends in An. arabiensis documented across this region [35-38].

Study results raised concerns pertaining to the comparability of WHO and CDC insecticide resistance tests. While in some areas, outcome measurements from both assays are reported to be equivalent in terms of resistance 


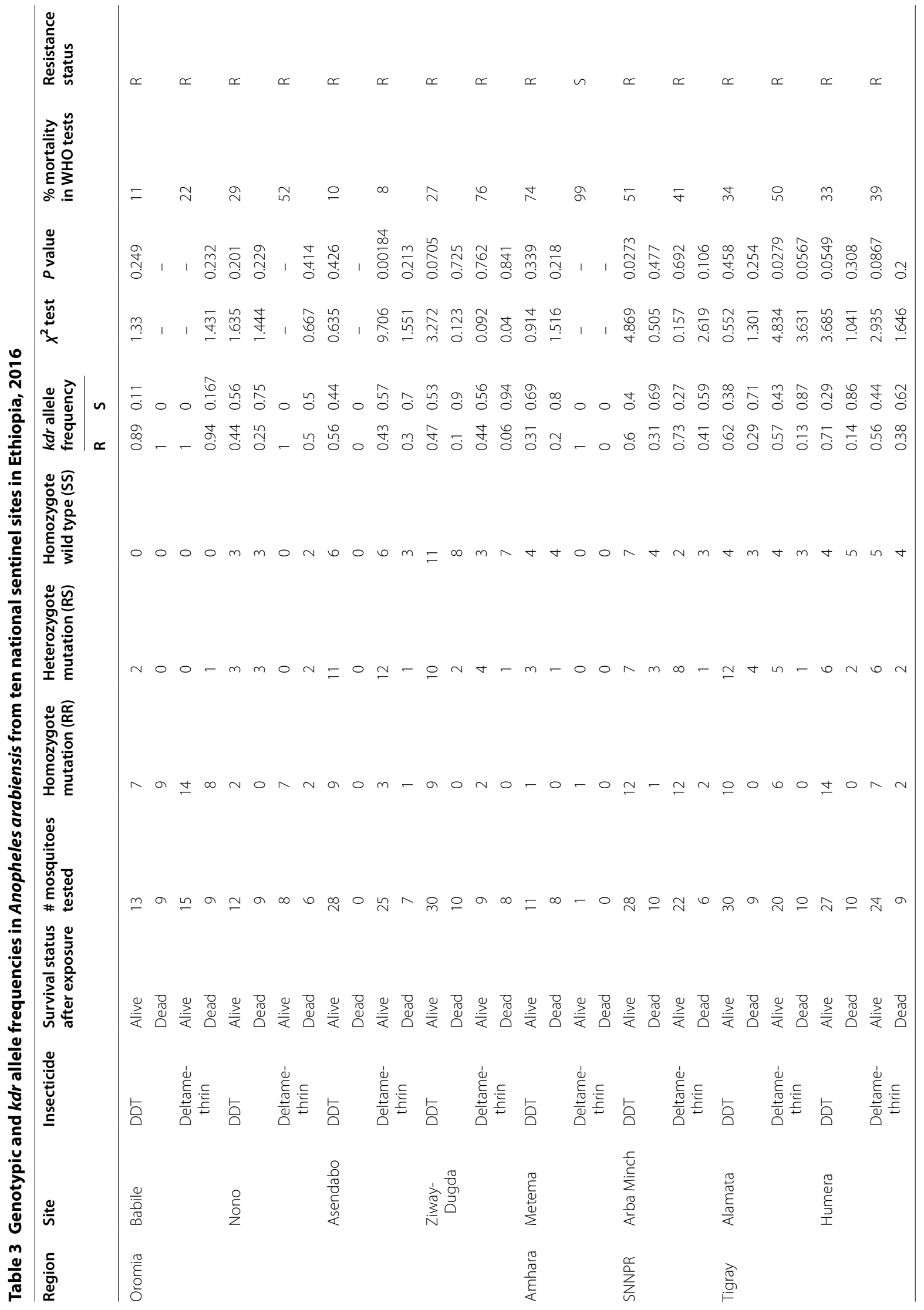




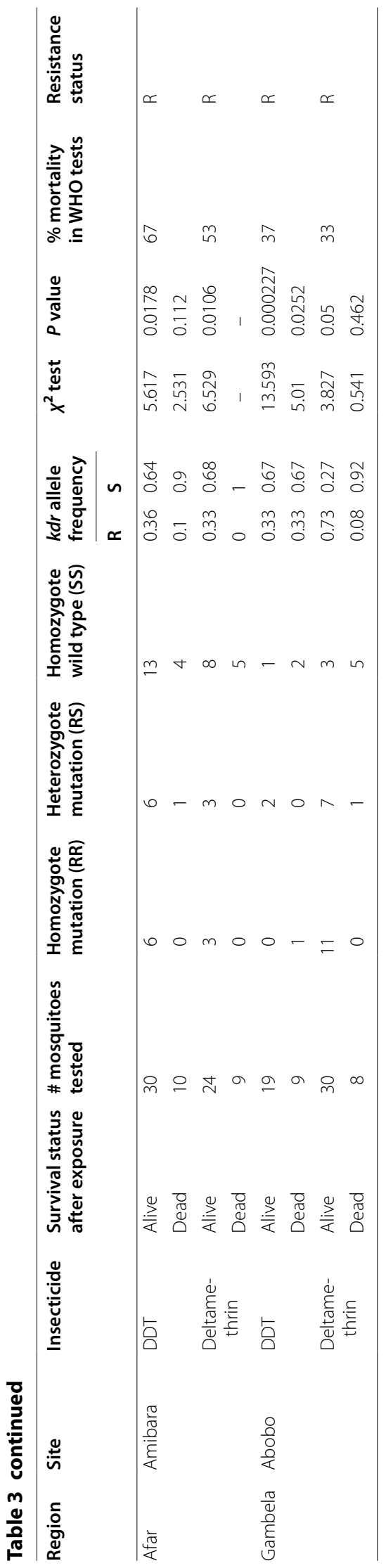


monitoring $[39,40]$, our data align with others reporting considerable discrepancies $[28,41,42]$, which were problematic to interpret, particularly when susceptibility profiles annually fluctuated above or below the thresholds of resistance set by the WHO $[18,28]$. Previous studies have suggested that the extent of inter-assay agreement may reflect levels of susceptibility heterogeneity, whereby tests conducted on vector populations with highly variable resistance profiles are more prone to inconsistent results [28]. Indeed, direct comparisons between our WHO and CDC bottle bioassays performed using bendiocarb and propoxur (where An. arabiensis were completely susceptible), and against pyrethroids (where resistance was more capricious) supports this supposition. Others have proposed that CDC bioassays may over-estimate pyrethroid resistance, as insecticide repellency can reduce the already relatively short contact time $(30 \mathrm{~min})$ of mosquitoes in coated bottles [40]. In this regard, our results demonstrated the opposite; higher and more uniform levels of pyrethroid resistance were obtained for WHO tests, when results were pooled across study sites.

There are a number of other technical and biological factors which could contribute to discordance between assays. WHO papers are distributed from a centralized source, which may render them prone to inter-batch variation and depending upon procurement schedules, can result in filter papers of different ages being used for the same monitoring activities in a given year. Ideally, to ensure consistency between study sites, all batches of papers would have been tested initially using a susceptible laboratory strain. CDC bottle bioassays are coated in-house which also introduces issues of standardization based on the proficiency of individual laboratory technicians, conditions of insecticide storage and numbers of consecutive times bottles are re-used [43-45]. Anopheles larvae were sampled from a range of the most productive breeding sites with different effective population sizes, genetic compositions, temperatures, nutritional access and chemical exposures depending upon local ecology. One important factor that was not investigated in this study was the influence that intensive agriculture pesticide use has had on resistance levels in Ethiopia (reviewed by [46]). Once collected, bioassays were conducted on emergent adult mosquitoes who were presumed to be An. arabiensis, based on PCR validation of a sub-set in 2015 and 2016. It should be noted that 10.4\% of all PCR reactions did not amplify either because of technical errors or the specimens did not belong to the species under investigation (An. gambiae s.s., An. arabiensis or An. quadriannulatus species $\mathrm{B} / A n$. amharicus). Finally, WHO tests assess mortality after a 24-h holding period, while CDC bioassays measure knock-down and acute toxicity, which depending upon the degree of vector tolerance, may not necessarily be interchangeable [28].

Regarding the underlying mechanisms of resistance, the restoration of pyrethroid susceptibility following preexposure to $\mathrm{PBO}$ and the non-association of L1014F $k d r$ allele frequency with levels of $A n$. arabiensis mortality in WHO bioassays, suggests that both over-expression of detoxification enzymes and target-site mutations are driving insecticide resistance. Moderate to high $k d r$ frequencies were detected in a number of sites and were fixed or approaching fixation in a minority. However, by comparison with earlier reports from the same areas, $k d r$ allele frequencies were lower overall $[9,14,47]$ and between our study years we also observed a slight, albeit not statistically significant, decline. In 2015, the lack of deviations from Hardy-Weinberg equilibrium in most areas indicated that selection for $k d r$ heterozygotes was not ongoing, allowing this allele to be lost to genetic drift in some populations, but by 2016, study sites from five regions demonstrated evidence for $k d r$ selection, potentially resulting from a mass distribution of LLINs by the NMCP beginning in August 2015. Given the lack of association between mosquito bioassay mortality and presence/absence of $k d r$ mutation, there are likely to be other mechanisms at play in the development of resistance. Future local surveillance programmes may wish to consider screening for additional, recently identified genetic markers of metabolic resistance in An. arabiensis, e.g. CYP6P4 [48].

\section{Conclusions}

To date, the presence of insensitive acetylcholinesterase mutations (ace- $1^{\mathrm{R}}$ or G119S), known to mediate resistance to both organophosphates and carbamates in $A n$. gambiae s.s. and An. arabiensis $[49,50]$, has not been reported in Ethiopia among vectors resistant to malathion or propoxur [9]. The bioassay results in this study were not indicative of any cross-resistance both between organophosphates and carbamates and among chemicals belonging to the same insecticide class. This observation suggests the existence of additional metabolic resistance mechanisms that can confer insecticide-specific resistance and also has implications for the development of an insecticide resistance management strategy. If interand intra-class rotation of different insecticides could be exploited to reduce selection pressures, this may have the potential to safeguard continued efficacy of IRS and other vector control strategies in Ethiopia and proactively mitigate the development of future insecticide resistance. Furthermore, additional epidemiological studies are warranted, in parallel with future resistance monitoring activities, to determine the operational impact of insecticide resistance on malaria vector control in Ethiopia. 


\section{Additional file}

Additional file 1: Tables S1. Summary of PMI-supported IRS activities in Ethiopia, 2008-2016. Table S2. Summary of insecticide resistance assays conducted per year in Ethiopia, 2012-2016. Table S3. Percentage corrected mortality (and numbers tested) of Anopheles arabiensis in WHO susceptibility tests conducted in Ethiopia, 2013. Table S4. Percentage corrected mortality (and numbers tested) of Anopheles arabiensis in WHO susceptibility tests conducted in Ethiopia, 2014. Table S5. Percentage corrected mortality (and numbers tested) of Anopheles arabiensis in WHO susceptibility tests conducted in Ethiopia, 2015. Table S6. Percentage corrected mortality (and numbers tested) of Anopheles arabiensis in WHO susceptibility tests conducted in Ethiopia, 2016. Table S7. Percentage corrected mortality (and numbers tested) of Anopheles arabiensis in CDC bottle bioassays conducted in Ethiopia, 2013. Table S8. Percentage corrected mortality (and numbers tested) of Anopheles arabiensis in CDC bottle bioassays conducted in Ethiopia, 2014. Table S9. Percentage corrected mortality (and numbers tested) of Anopheles arabiensis in CDC bottle bioassays conducted in Ethiopia, 2015. Table S10. Percentage corrected mortality (and numbers tested) of Anopheles arabiensis in CDC bottle bioassays conducted in Ethiopia, 2016. Table S11. Percentage corrected mortality (and numbers tested) of Anopheles arabiensis in WHO susceptibility tests and CDC bottle bioassays conducted in Ziway Dugda Ethiopia, 2013-2016.

\section{Authors' contributions}

JS, SRI, YY, SC, DD, HS, KG, RAW and DY designed the study and were responsible for data analysis and interpretation. JS, GYA, AG, YY, EK, EZ, AA, AS, and DY led the entomology field activities and laboratory assays and participated in data collection. SRI, JS, GD, SC, YY, DD, RAW, CF, and DD were responsible for project oversight and management. LAM, SRI and DY drafted the manuscript which was revised by co-authors. All authors read and approved the final manuscript.

\section{Author details}

1 Entomology Branch, Centers for Disease Control and Prevention, 1600 Clifton Road, Atlanta, GA 30329-4027, USA. ${ }^{2}$ President's Malaria Initiative Africa Indoor Residual Spraying Project, Abt Associates, Gerji Road, Sami Building, 1st Floor, Addis Ababa, Ethiopia. ${ }^{3}$ U.S. Agency for International Development (USAID), Entoto Street, Addis Ababa, Ethiopia. ${ }^{4}$ President's Malaria Initiative Africa Indoor Residual Spraying Project, Abt Associates, 4550 Montgomery Ave., Suite 800 North, Bethesda, MD 20814, USA. ${ }^{5}$ Tropical and Infectious Diseases Research Center, Jimma University, Jimma, Ethiopia. ${ }^{6}$ Medical and Entomology Unit, Institute of Bio-Medical Sciences, College of Health Sciences, Mekelle University, Mek'ele, Ethiopia. ${ }^{7}$ National Malaria Control Programne, Federal Ministry of Health, Addis Ababa, Ethiopia. ${ }^{8}$ President's Malaria Initiative, United States Agency for International Development, Bureau for Global Health, Office of Infectious Disease, 2100 Crystal Drive, Arlington, VA 22202, USA. ${ }^{9}$ Department of Medical Laboratory Sciences and Pathology, College of Health Sciences, Jimma University, Jimma, Ethiopia.

\section{Acknowledgements}

The authors would like to thank all mosquito collectors for their dedicated work and William Brogdon and Matthew Murphy for their technical assistance and support. This study was conducted through the United States Agency for International Development (USAID) funded Presidential Malaria Initiative (PMI) Africa Indoor Residual Spraying Project (AIRS) Project. The opinions expressed herein are those of the authors and do not necessarily reflect the views of USAID.

\section{Competing interests}

The authors declare that they have no competing interests.

\section{Availability of data and materials}

Not applicable.

\section{Consent for publication}

Not applicable.

\section{Ethics approval and consent to participate}

The study protocol was reviewed and approved by the institutional review board (IRB) of the College of Health Sciences at Jimma University.

\section{Funding}

This study was conducted by the United States Agency for International Development's (USAID) Africa Indoors Residual Spraying Project (AIRS), funded by the US President's Malaria Initiative (PMI). LAM is supported through an American Society for Microbiology Fellowship at the CDC.

\section{Publisher's Note}

Springer Nature remains neutral with regard to jurisdictional claims in published maps and institutional affiliations.

Received: 1 July 2017 Accepted: 10 November 2017

Published online: 18 November 2017

\section{References}

1. WHO. World Malaria Report 2016. Geneva: World Health Organization; 2016.

2. President's Malaria Initiative. Malaria Operational Plan FY 2016-Ethiopia. USAID; 2016.

3. Africa Indoor Residual Spraying Project, A.A.I. Ethiopia: Entomological Monitoring of 2015 IRS Activities. Final Report. Addis Ababa, Ethiopia; 2015.

4. MOH. National malaria strategic plan (2014-2020). Addis Ababa: Federal Democratic Republic of Ethiopia, Ministry of Health; 2014.

5. Abose T, Yeebiyo Y, Olana D, Alamirew D, Beyene Y, Regassa L, et al. Reorientation and definition of the role of malaria vector control in Ethiopia. The epidemiology and control of malaria with special emphasis on the distribution, behaviour and susceptibility of insecticides of Anopheline vectors and chloroquine resistance in Zwai, central Ethiopia and other areas. WHO/MAL/98.1085; 1998

6. Nigatu W, Petros B, Lulu M, Adunga N, Wirtz R, Tilahun D. Some aspects of malaria prevalence, vector infectivity and DDT resistance studies in Gambella Region, South Western Ethiopia. Ethiop J Health Dev. 1994;8:1-10.

7. Balkew M, Gebre-Michael T, Hailu A. Insecticide susceptibility level of Anopheles arabiensis in two agro-development localities in eastern Ethiopia. Parassitologia. 2003:45:1-3.

8. Balkew M, Ibrahim M, Koekemoer LL, Brooke BD, Engers H, Aseffa A, et al. Insecticide resistance in Anopheles arabiensis (Diptera: Culicidae) from villages in central, northern and south west Ethiopia and detection of $k d r$ mutation. Parasites Vectors. 2010;3:40.

9. Yewhalaw D, Wassie F, Steurbaut W, Spanoghe P, Van Bortel W, Denis L, et al. Multiple insecticide resistance: an impediment to insecticide-based malaria vector control program. PLoS ONE. 2011;6:e16066.

10. Asale A, Getachew Y, Hailesilassie W, Speybroeck N, Duchateau L, Yewhalaw D. Evaluation of the efficacy of DDT indoor residual spraying and long-lasting insecticidal nets against insecticide resistant populations of Anopheles arabiensis Patton (Diptera: Culicidae) from Ethiopia using experimental huts. Parasites Vectors. 2014;7:131.

11. Abate A, Hadis M. Susceptibility of Anopheles gambiae s.l. to DDT, malathion, permethrin and deltamethrin in Ethiopia. Trop Med Int Health. 2011;16:486-91.

12. Yewhalaw D, Asale A, Tushune K, Getachew Y, Duchateau L, Speybroeck N. Bio-efficacy of selected long-lasting insecticidal nets against pyrethroid resistant Anopheles arabiensis from South-Western Ethiopia. Parasites Vectors. 2012;5:159.

13. Massebo F, Balkew M, Gebre-Michael T, Lindtørn B. Blood meal origins and insecticide susceptibility of Anopheles arabiensis from Chano in South-West Ethiopia. Parasites Vectors. 2013;6:44.

14. Fettene M, Olana D, Christian RN, Koekemoer LL, Coetzee M. Insecticide resistance in Anopheles arabiensis from Ethiopia. Afr Entomol. 2013;21:89-94. 
15. Balkew M, Getachew A, Chibsa S, Olana D, Reithinger R, Brogdon W. Insecticide resistance: a challenge to malaria vector control in Ethiopia. Malar J. 2012;11:139.

16. $\mathrm{FMoH}$. Insecticide resistance monitoring and management strategy for malaria vector control in Ethiopia. Disease prevention and control directorate. National malaria control and elimination programme. Ethiopia; 2016

17. WHO. Test procedures for insecticide resistance monitoring in malaria vector mosquitoes. Geneva: World Health Organization; 2013.

18. WHO. Test procedures for insecticide resistance monitoring in malaria vectors, bio-efficacy and persistence of insecticides on treated surfaces. Report of the WHO informal consultation 28-30 September 1998. WHO CDS/MAL/98.12; 1998.

19. Brogdon W, Chan A. Guideline for evaluating insecticide resistance in vectors using the CDC bottle bioassay. https://www.cdc.gov/malaria/ resources/pdf/fsp/ir_manual/ir_cdc_bioassay_en.pdf. 2013.

20. Gillies T, Coetzee M. Supplement of the Anopheles of Africa south of Sahara (Afrotropical region) Johannesburg: Republic of South Africa. Publication of the South African Institute of Medical Research; 1987.

21. Collins FH, Mendez MA, Rasmussen MO, Mehaffey PC, Besansky NJ, Finnerty $V$. A ribosomal RNA gene probe differentiates member species of the Anopheles gambiae complex. Am J Trop Med Hyg. 1987;37:37-41.

22. Scott JA, Brogdon WG, Collins FH. Identification of single specimens of the Anopheles gambiae complex by the polymerase chain reaction. Am J Trop Med Hyg. 1993;49:520-9.

23. Fettene M, Temu EA. Species-specific primer for identification of Anopheles quadriannulatus sp. B (Diptera: Culicidae) from Ethiopia using a multiplex polymerase chain reaction assay. J Med Entomol. 2003;40:112-5.

24. Verhaeghen K, Van Bortel W, Roelants P, Backeljau T, Coosemans M. (Detection of the East and West African kdr mutation in Anopheles gambiae and Anopheles arabiensis from Uganda using a new assay based on FRET/Melt curve analysis. Malar J. 2006;5:16.

25. Martinez-Torres D, Chandre F, Williamson MS, Darriet F, Berge JB, Devonshire AL, et al. Molecular characterization of pyrethroid knockdown resistance $(k d r)$ in the major malaria vectors Anopheles gambiae s.s. Insect Mol Biol. 1998;7:179-84.

26. Ranson H, Jensen B, Vulule J, Wang X, Hemingway J, Collins FH. Identification of point mutation in the voltage-gated sodium channel gene of Kenyan Anopheles gambiae associated with resistance to DDT and pyrethroids. Insect Mol Biol. 2000;9:491-7.

27. Abbott WS. A method of computing the effectiveness of insecticide. $J$ Econ Entomol. 1925:18:265-7.

28. Owusu HF, Jancaryova D, Malone D, Müller P. Comparability between insecticide resistance bioassays for mosquito vectors: time to review current methodology? Parasites Vectors. 2015;8:357.

29. Viera AJ, Garrett JM. Understanding interobserver agreement: the kappa statistic. Fam Med. 2005;37:360-3.

30. Strode C, Donegan S, Garner P, Enayati AA, Hemingway J. The impact of pyrethroid resistance on the efficacy of insecticide-treated bed nets against African Anopheline mosquitoes: systematic review and metaanalysis. PLoS Med. 2014;11:e1001619.

31. Riveron JM, Chiumia M, Menze BD, Barnes KG, Irving H, Ibrahim SS, et al. Rise of multiple insecticide resistance in Anopheles funestus in Malawi: a major concern for malaria vector control. Malar J. 2015;14:344

32. Sadasivaiah S, Tozan Y, Breman JG. Dichlorodiphenyltrichloroethane (DDT) for indoor residual spraying in Africa: how can it be used for malaria control? Am J Trop Med Hyg. 2007;77:249-63.

33. Yeebiyo Y, Dengela D, Tesfaye AG, Anshebo GY, Kolyada L, Wirtz R, et al. Short persistence of bendiocarb sprayed on previous walls and its implications for the indoor residual spray program in Ethiopia. Parasites Vectors. 2016;9:266

34. Ranson $\mathrm{H}$, Abdallah $\mathrm{H}$, Badolo A, Guelbeogo WM, Kerah-Hinzoumbé C, Yangalbé-Kalnoné E, Sagnon N, et al. Insecticide resistance in Anopheles gambiae: data from the first year of a multi-country study highlight the extent of the problem. Malar J. 2009;8:299.

35. Deressa W, Loha E, Balkew M, Hailu A, Gari T, Kenea O, et al. Combining long-lasting insecticidal nets and indoor residual spraying for malaria prevention in Ethiopia: study protocol for a cluster randomized controlled trial. Trials. 2016;17:20

36. Abdalla H, Wilding CS, Nardini L, Pignatelli P, Koekemoer LL, Ranson H, et al. Insecticide resistance in Anopheles arabiensis in Sudan: temporal trends and underlying mechanisms. Parasites Vectors. 2014;7:213.

37. Abuelmaali SA, Elaagip AH, Basheer MA, Frah EA, Ahmed FTA, Elhaj HFA, et al. Impacts of agricultural practices on insecticide resistance in the malaria vector Anopheles arabiensis in Khartoum State, Sudan. PLoS ONE. 2013;8:e80549

38. Wanjala CL, Mbugi JP, Ototo E, Gesuge M, Afrane YA, Atieli HE, et al. Pyrethroid and DDT resistance and organophosphate susceptibility among Anopheles spp. mosquitoes, Western Kenya. Emerg Infect Dis. 2014;21:2178-81.

39. $\mathrm{MoH}$. Malaria Vector Control Situation Analysis. National Malaria Control Programme. Ministry of Health. The State of Eritrea; 2014.

40. Aïzoun N, Osse R, Azondekon R, Alia R, Oussou O, Gnanguenon V, et al. Comparison of the standard WHO susceptibility tests and the CDC bottle bioassay for the determination of insecticide susceptibility in malaria vector and their correlation with biochemical and molecular biology assays in Benin, West Africa. Parasites Vectors. 2013;6:147.

41. Ochomo E, Bayoh MN, Brogdon WG, Gimnig JE, Ouma C, Vulule JM, et al. Pyrethroid resistance in Anopheles gambiae s.s. and Anopheles arabiensis in western Kenya: phenotypic, metabolic and target site characterizations of three populations. Med Vet Entomol. 2013;27:156-64.

42. Fonseca-Gonzalez I, Cardenas R, Quiñones ML, McAllister J, Brogdon WG. Pyrethroid and organophosphates resistance in Anopheles (N.) nuneztovari Gabaldón populations from malaria endemic areas in Colombia. Parasitol Res. 2009;105:1399-409.

43. Perea EZ, León RB, Salcedo MP, Brogdon WG, Devine GJ. Adaptation and evaluation of the bottle bioassay for monitoring insecticide resistance in disease vector mosquitoes in the Peruvian Amazon. Malar J. 2009;8:208.

44. Aïzoun N, Azondekon R, Aïkpon R, Gnanguenon V, Osse R, Asidi A, et al. Study of the efficacy of a Wheaton coated bottle with permethrin and deltamethrin in laboratory conditions and a WHO impregnated paper with bendiocarb in field conditions. Asian Pac J Trop Biomed. 2014:4:492-7.

45. Aïzoun N, Azondekon R, Akogbéto M. Determination of the days storage and re-use of a Wheaton coated bottle with DDT, fenitrothion, and bendiocarb in laboratory conditions. Int J Curr Microbiol App Sci. 2014:3:203-10.

46. Reid MC, McKenzie FE. The contribution of agricultural insecticide use to increasing insecticide resistance in African malaria vectors. Malar J. 2016;15:107.

47. Yewhalaw D, Bortel WV, Denis L, Coosemans M, Duchateau L, Speybroeck $N$. First evidence of high knockdown resistance frequency in Anopheles arabiensis (Diptera: Culicidae) in Ethiopia. Am J Trop Med Hyg. 2010;83:122-5.

48. Ibrahim SS, Riverson JM, Stott R, Irving H, Wondji CS. The cytochrome P450 CYP6P4 is responsible for the high pyrethroid resistance in knockdown resistance-free Anopheles arabiensis. Insect Biochem Mol Biol. 2016:68:23-32.

49. Djogbénou L, Dabiré R, Diabaté $A$, Kengne $P$, Akogbéto JM, Chandre F. Identification and geographic distribution of the $A C E-1^{R}$ mutation in the malaria vector Anopheles gambiae in South-Western Burkina Faso, West Africa. Am J Trop Med Hyg. 2008;78:298-302.

50. Dabiré RK, Namountougou M, Diabaté A, Soma DD, Bado J, Toé HK, et al. Distribution and frequency of kdr mutations within Anopheles gambiae s.l. populations and first report of the Ace. 1G119S mutation in Anopheles arabiensis from Burkina Faso (West Africa). PLoS ONE. 2014;9:e101484. 